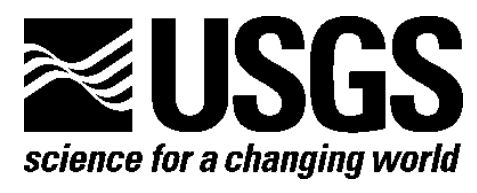

\title{
Vitrinite Reflectance Data for Cretaceous Marine Shales and Coals in the Bighorn Basin, North-Central Wyoming and South-Central Montana
}

By Mark J. Pawlewicz and Thomas M. Finn

Open-File Report 2012-1254

U.S. Department of the Interior

U.S. Geological Survey 


\section{U.S. Department of the Interior \\ KEN SALAZAR, Secretary}

\section{U.S. Geological Survey \\ Marcia K. McNutt, Director}

U.S. Geological Survey, Reston, Virginia: 2012

For more information on the USGS-the Federal source for science about the Earth, its natural and living resources, natural hazards, and the environment-visit http://www.usgs.gov or call 1-888-ASK-USGS

For an overview of USGS information products, including maps, imagery, and publications, visit http://www.usgs.gov/pubprod

To order this and other USGS information products, visit http://store.usgs.gov

Suggested citation:

Pawlewicz, M.J., and Finn, T.M., 2012, Vitrinite reflectance data for Cretaceous marine shales and coals in the Bighorn Basin, north-central Wyoming and south-central Montana: U.S. Geological Survey Open-File Report 2012-1254, 11 p.

Any use of trade, product, or firm names is for descriptive purposes only and does not imply endorsement by the U.S. Government.

Although this report is in the public domain, permission must be secured from the individual copyright owners to reproduce any copyrighted material contained within this report. 


\section{Contents}

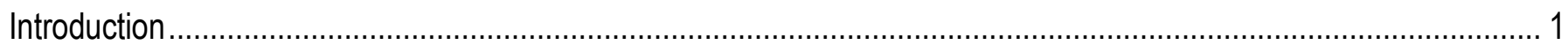

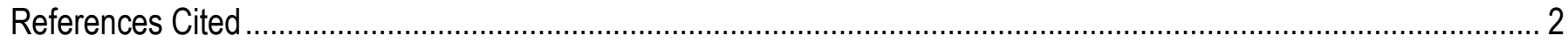

\section{Figures}

1. Map of the Rocky Mountain region showing Laramide basins ..................................................................... 4

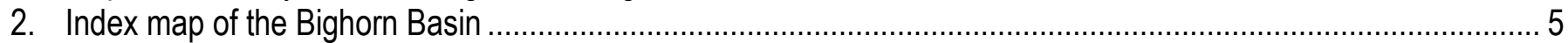

3. Stratigraphic chart of uppermost Jurassic to lower Tertiary rocks in the Bighorn Basin ...................................... 6

\section{Table}

1. Vitrinite reflectance data for the Bighorn Basin, Wyoming ........................................................................ 


\title{
Vitrinite Reflectance Data for Cretaceous Marine Shales and Coals in the Bighorn Basin, North-Central Wyoming and South-Central Montana
}

\author{
By Mark J. Pawlewicz and Thomas M. Finn
}

\section{Introduction}

The Bighorn Basin is a large Laramide (Late Cretaceous through Eocene) structural and sedimentary basin that encompasses about 10,400 square miles in north-central Wyoming and southcentral Montana (fig. 1). The basin is bounded on the northeast by the Pryor Mountains, on the east by the Bighorn Mountains, and on the south by the Owl Creek Mountains (fig. 2). The north boundary, as defined in this report, includes a zone of faulting and folding referred to as the Nye-Bowler lineament (Wilson, 1936). The northwest and west margins are formed by the Beartooth Mountains and Absaroka Range, respectively (fig. 2).

Important conventional oil and gas resources have been discovered and produced from reservoirs ranging in age from Cambrian through Tertiary (Fox and Dolton, 1989; 1996a, b; De Bruin, 1993). In addition, a potential unconventional basin-centered gas accumulation may be present in Cretaceous reservoirs (Johnson and Finn, 1998; Johnson and others, 1999; Surdam and others, 1997; Finn and others, 2010). In recent years, advances and success in horizontal drilling and multi-stage fracture stimulation have led to an increase in exploration and completion of wells in marine shales in other Rocky Mountain Laramide basins that were traditionally thought of as hydrocarbon source rocks. Important parameters that control hydrocarbon production from these shales include: reservoir thickness, amount and type of organic matter, and thermal maturity (Milici, 1993; Curtis, 2002). The purpose of this report is to present new vitrinite reflectance data collected from Cretaceous marine shales and coals in the Bighorn Basin to better characterize the thermal maturity and petroleum potential of these rocks. These new data supplement previously published data by Hagen (1986), Nuccio and Finn (1998), Yin (1997), and Finn and Pawlewicz (2007).

Ninety-eight samples from Lower Cretaceous and lowermost Upper Cretaceous strata (fig. 3) were collected from well cuttings from wells stored at the U.S. Geological Survey (USGS) Core Research Center in Lakewood, Colorado. Sample locations are shown on figure 2. All samples were analyzed by vitrinite reflectance to determine levels of thermal maturation. Preparation of samples for reflectance analysis required (1) crushing the larger pieces into 0.25- to 1-millimeter pieces, (2) casting the pieces with epoxy in pre-cut and drilled plugs, and (3) curing the samples overnight. Subsequently, a four-step grinding and polishing process was implemented that included sanding with progressively finer sandpaper (60 to 600 grit) followed by a two-step polishing process ( 0.3 and 0.05 micron). Vitrinite reflectance measurements were determined at $500 \mathrm{X}$ magnification using plane-polarized incident white light and a 546-nanometer monochromatic filter in immersion oil. For samples containing sufficiently high quality vitrinite, at least 25 measurements were recorded. For samples of poorer quality, either due to a poor polish or to the presence of mineral or other inorganic material, fewer measurements were recorded. Analytical results are given in table 1. 


\section{References Cited}

Curtis, J.B., 2002, Fractured shale-gas systems: American Association of Petroleum Geologists Bulletin, v. 86 , no. 11 , p. $1,921-1,938$.

De Bruin, R.H., 1993, Overview of oil and gas geology of Wyoming, in Snoke, A.W., Steidtmann, J.R., and Roberts, S.M., eds., Geology of Wyoming: Geological Survey of Wyoming Memoir No. 5, p. 836-873.

Dickinson, W.R., Klute, M.A., Hayes, M.J., Janecke, S.U., Lundin, E.R., McKittrick, M.A., and Olivares, M.D., 1988, Paleographic and paleotectonic setting of Laramide sedimentary basins in the central Rocky Mountain region: Geological Society of America Bulletin, v. 100, p. 1,023-1,039.

Finn, T.M., and Pawlewicz, M.J., 2007, New vitrinite reflectance data for the Bighorn Basin, northcentral Wyoming and south-central Montana: U.S. Geological Survey Open-File Report 2007-1246, $4 \mathrm{p}$.

Finn, T.M., Kirschbaum, M.A., Roberts, S.B., Condon, S.M., Roberts, L.N.R., and Johnson, R.C., 2010, Cretaceous-Tertiary Composite Total Petroleum System (503402), Bighorn Basin, Wyoming and Montana, Petroleum Systems and Geologic Assessment of Oil and Gas Resources in the Bighorn Basin Province, Wyoming and Montana: U.S. Geological Survey Digital Data Series DDS-69-V, chap. 3, 146 p., CD-ROM.

Fox, J.E., and Dolton, G.L., 1989, Petroleum geology of the Wind River and Bighorn Basins, Wyoming and Montana: U.S. Geological Survey Open-File Report 87-450P, 41 p.

Fox, J.E., and Dolton, G.L., 1996a, Bighorn Basin Province, in Gautier, D.L., Dolton, G.L., Takahashi, K.I., and Varnes, K.L., eds., National assessment of United States oil and gas resources-Results, methodology, and supporting data: U.S. Geological Survey Digital Data Series DDS-30, Release 2, CD-ROM.

Fox, J.E., and Dolton, G.L., 1996b, Petroleum geology of the Bighorn Basin, north-central Wyoming and south-central Montana, in Bowen, C.E., Kirkwood, S.C., and Miller, T.S., eds., Resources of the Bighorn Basin: Wyoming Geological Association Guidebook, p. 19-39.

Green, G.N., and Drouillard, P.H., 1994, The digital geologic map of Wyoming in ARC/INFO format: U.S. Geological Survey Open-File Report 94-425, scale 1:500,000.

Hagen, E.S., 1986, Hydrocarbon maturation in Laramide-style basins - Constraints from the northern Bighorn Basin, Wyoming and Montana: Laramie, University of Wyoming, Ph.D. dissertation, 215 p. Johnson, R.C., and Finn, T.M., 1998, Is there a basin-centered gas accumulation in Upper Cretaceous rocks in the Bighorn Basin?, in Keefer, W.R., and Goolsby, J.E., eds., Cretaceous and Lower Tertiary rocks of the Bighorn Basin, Wyoming and Montana: Wyoming Geological Association Guidebook, p. 257-273.

Johnson, R.C., Crovelli, R.A., Lowell, B.G., and Finn, T.M., 1999, An assessment of in-place gas resources in the low-permeability basin-centered gas accumulation of the Bighorn Basin, Wyoming and Montana: U.S. Geological Survey Open-File Report 99-315-A, 123 p.

Milici, R.C., 1993, Autogenic gas (self sourced) from shales-An example from the Appalachian Basin: U.S. Geological Professional Paper 1570, p. 253-278.

Nuccio, V.F., and Finn, T.M., 1998, Thermal maturity and petroleum generation history of Cretaceous and Tertiary source rocks, Bighorn Basin, Wyoming and Montana, in Keefer, W.R., and Goolsby, J.E., eds., Cretaceous and Lower Tertiary rocks of the Bighorn Basin, Wyoming and Montana: Wyoming Geological Association Guidebook, p. 211-231.

Raines, G.L., and Johnson, B.R., 1995, Digital representation of the Montana state geologic map-A contribution to the Interior Colombia River basin ecosystem management project: U.S. Geological Survey Open-File Report 95-691, 20 p. 
Surdam, R.C., Jiao, Z.S., and Heasler, H.P., 1997, Anomalously pressured gas compartments in Cretaceous rocks of the Laramide basins of Wyoming - A new class of hydrocarbon accumulation, in Surdam, R.C., ed., Seals, traps, and the petroleum system: American Association of Petroleum Geologists Memoir 67, p. 199-222.

Wilson, C.W., Jr., 1936, Geology of the Nye-Bowler lineament, Stillwater and Carbon Counties, Montana: American Association of Petroleum Geologists Bulletin, v. 20, no. 9, p. 1,161-1,188.

Yin, Peigui, 1997, Source rock maturation and diagenetic modeling in Badger Basin, in Campen, E.R., ed., Bighorn Basin-50 Years on the Frontier, Part 2: Yellowstone Bighorn Research AssociationWyoming Geological Association-Montana Geological Association, 1997 Field Trip and Symposium, p. 41-44. 


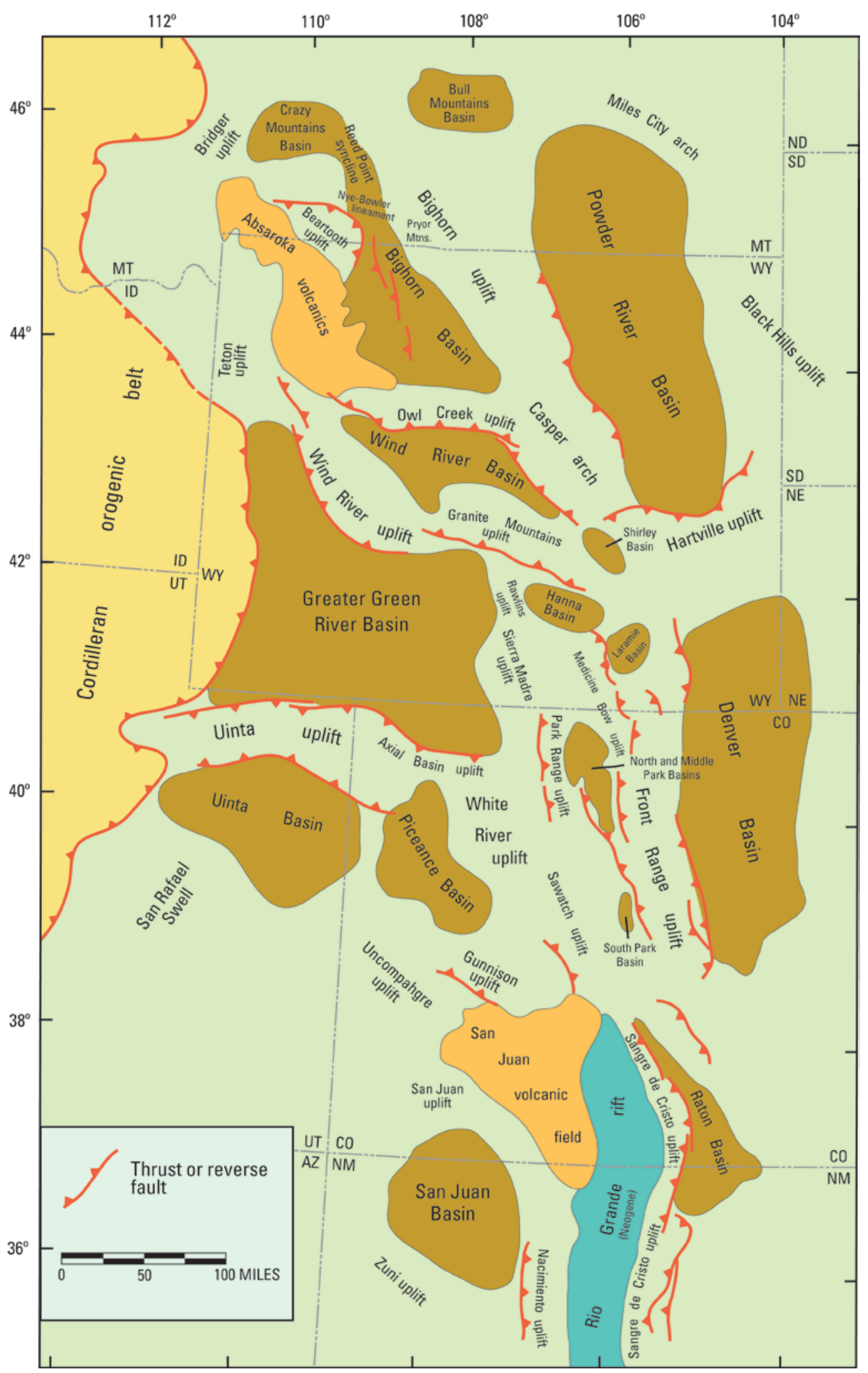

Figure 1. Map of the Rocky Mountain region extending from southern Montana to northern New Mexico showing locations of Laramide sedimentary and structural basins and intervening uplifts. Modified from Dickinson and others (1988). 


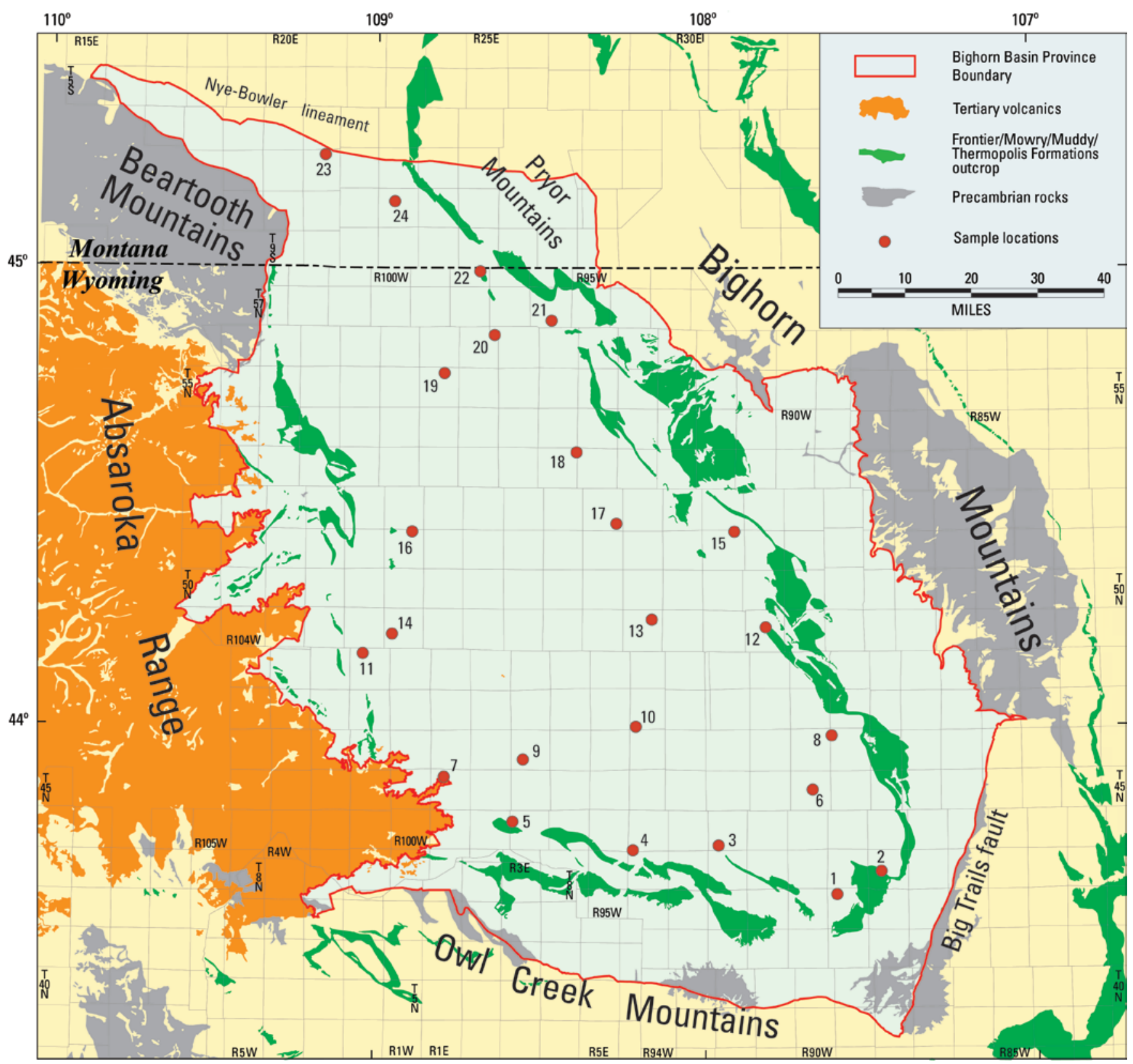

Figure 2. Map showing general outline of the Bighorn Basin, bordering mountain ranges, distribution of major rock units, and sample localities. Province boundary from Finn and others (2010). Outcrops from Green and Drouillard (1994), and Raines and Johnson (1995). Numbers refer to column 1 (location) in table 1. 


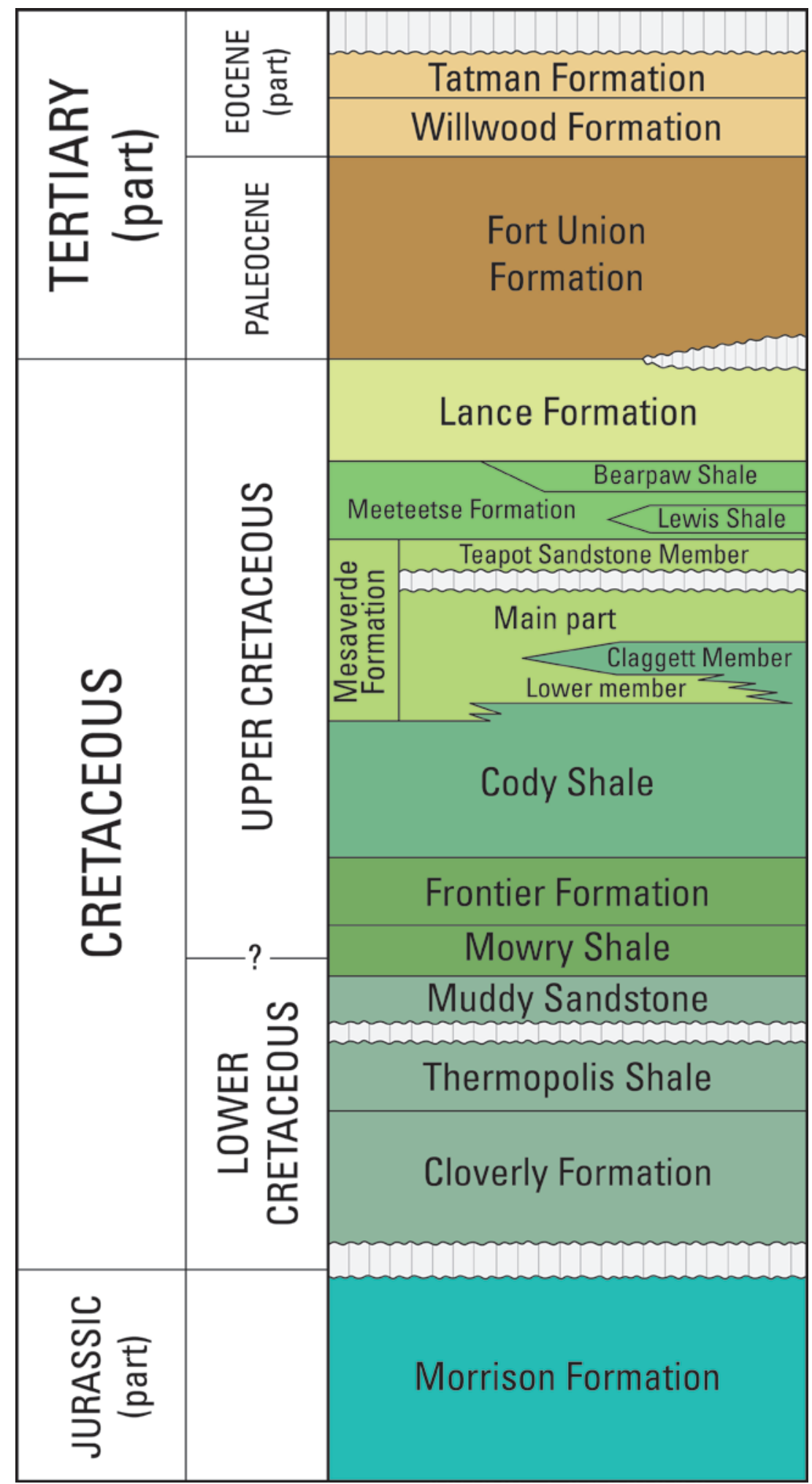

Figure 3. Generalized stratigraphic chart of uppermost Jurassic, Cretaceous, and lower Tertiary rocks in the Bighorn Basin. 
Table 1. Vitrinite reflectance (Ro) data and locations for cuttings samples from wells drilled for oil and gas exploration and production in the Bighorn Basin, north-central Wyoming and south-central Montana. Map no. refers to sample location numbers shown on figure 2.

[API, well number assigned by American Petroleum Institute; Tnsp., township; Rng., range; Sec., section; Lith., lithology; \%Ro, vitrinite reflectance measurement; n, number of readings per sample; nr, no reading. Queried (?) where uncertain. Depth is in feet.]

\begin{tabular}{|c|c|c|c|c|c|c|c|c|c|c|c|c|}
\hline $\begin{array}{c}\text { Map } \\
\text { no. }\end{array}$ & API & Tnsp. & Rng. & Sec. & Operator & Well & $\begin{array}{c}\text { Top } \\
\text { depth }\end{array}$ & $\begin{array}{l}\text { Bottom } \\
\text { depth }\end{array}$ & Formation & Lith. & $\% R_{0}$ & $\mathbf{n}$ \\
\hline 1 & 49043050090000 & $42 \mathrm{~N}$ & $89 W$ & 5 & Superior Oil & 24-5 Unit Orchard & 550 & 560 & Cody & shale & 0.49 & 2 \\
\hline 1 & 49043050090000 & $42 \mathrm{~N}$ & $89 \mathrm{~W}$ & 5 & Superior Oil & 24-5 Unit Orchard & 1,070 & 1,080 & Frontier & shale & 0.60 & 3 \\
\hline 1 & 49043050090000 & $42 \mathrm{~N}$ & $89 W$ & 5 & Superior Oil & 24-5 Unit Orchard & 1,820 & 1,830 & Mowry & shale & 0.61 & 6 \\
\hline 1 & 49043050090000 & $42 \mathrm{~N}$ & $89 W$ & 5 & Superior Oil & 24-5 Unit Orchard & 2,050 & 2,060 & Thermopolis & shale & 0.67 & 1 \\
\hline 2 & 49043202510000 & $43 N$ & $88 W$ & 20 & Ashland Exploration & 1-20 Chabot-Govt. & 300 & 360 & Frontier & shale & 0.45 & 4 \\
\hline 2 & 49043202510000 & $43 N$ & $88 W$ & 20 & Ashland Exploration & 1-20 Chabot-Govt. & 540 & 570 & Mowry & shale & $\mathrm{nr}$ & \\
\hline 2 & 49043202510000 & $43 N$ & $88 W$ & 20 & Ashland Exploration & 1-20 Chabot-Govt. & 720 & 750 & Mowry & shale & $\mathrm{nr}$ & \\
\hline 2 & 49043202510000 & $43 N$ & $88 \mathrm{~W}$ & 20 & Ashland Exploration & 1-20 Chabot-Govt. & 940 & 970 & Thermopolis & shale & 0.75 & 6 \\
\hline 3 & 49043050610000 & $44 \mathrm{~N}$ & $92 \mathrm{~W}$ & 32 & Continental Oil & 1 Govt. Mann & 360 & 370 & Cody & shale & 0.59 & 9 \\
\hline 3 & 49043050610000 & $44 \mathrm{~N}$ & $92 \mathrm{~W}$ & 32 & Continental Oil & 1 Govt. Mann & 1,080 & 1,090 & Frontier & shale & 0.73 & 11 \\
\hline 3 & 49043050610000 & $44 N$ & $92 \mathrm{~W}$ & 32 & Continental Oil & 1 Govt. Mann & 1,740 & 1,750 & Mowry & shale & 0.73 & 11 \\
\hline 3 & 49043050610000 & $44 N$ & $92 \mathrm{~W}$ & 32 & Continental Oil & 1 Govt. Mann & 2,010 & 2,020 & Thermopolis & shale & 0.97 & 9 \\
\hline 4 & 49017200830000 & $44 N$ & $94 \mathrm{~W}$ & 31 & Bagdad Oil & 1 Lyke & 250 & 280 & Cody & shale & 0.54 & 8 \\
\hline 4 & 49017200830000 & $44 N$ & $94 \mathrm{~W}$ & 31 & Bagdad Oil & 1 Lyke & 1,330 & 1,360 & Mowry & shale & 0.55 & 1 \\
\hline 4 & 49017200830000 & $44 N$ & $94 \mathrm{~W}$ & 31 & Bagdad Oil & 1 Lyke & 1,600 & 1,630 & Thermopolis & shale & 0.37 & 1 \\
\hline 5 & 49017058630000 & $44 \mathrm{~N}$ & $97 \mathrm{~W}$ & 7 & Westates Petroleum & 2-7 Lewis & 720 & 740 & Frontier & shale & 0.64 & 4 \\
\hline 5 & 49017058630000 & $44 \mathrm{~N}$ & $97 \mathrm{~W}$ & 7 & Westates Petroleum & 2-7 Lewis & 1,040 & 1,080 & Mowry & shale & $\mathrm{nr}$ & \\
\hline 5 & 49017058630000 & $44 N$ & $97 \mathrm{~W}$ & 7 & Westates Petroleum & 2-7 Lewis & 1,200 & 1,220 & Mowry & shale & 0.67 & 7 \\
\hline 5 & 49017058630000 & $44 \mathrm{~N}$ & $97 \mathrm{~W}$ & 7 & Westates Petroleum & 2-7 Lewis & 1,450 & 1,470 & Thermopolis & shale & $\mathrm{nr}$ & \\
\hline
\end{tabular}


Table 1. Vitrinite reflectance (Ro) data and locations for cuttings samples from wells drilled for oil and gas exploration and production in the Bighorn

Basin, north-central Wyoming and south-central Montana. Map no. refers to sample location numbers shown on figure 2.-Continued

[API, well number assigned by American Petroleum Institute; Tnsp., township; Rng., range; Sec., section; Lith., lithology; \%Ro, vitrinite reflectance measurement; n, number of readings per sample; nr, no reading. Queried (?) where uncertain. Depth is in feet.]

\begin{tabular}{|c|c|c|c|c|c|c|c|c|c|c|c|c|}
\hline $\begin{array}{l}\text { Map } \\
\text { no. }\end{array}$ & API & Tnsp. & Rng. & Sec. & Operator & Well & $\begin{array}{l}\text { Top } \\
\text { depth }\end{array}$ & $\begin{array}{c}\text { Bottom } \\
\text { depth }\end{array}$ & Formation & Lith. & $\% R_{\circ}$ & $\mathbf{n}$ \\
\hline 6 & 49043205120000 & $45 \mathrm{~N}$ & $90 \mathrm{~W}$ & 15 & Pike Resources & 1-15 Federal & 4,780 & 4,810 & Cody & shale & 0.86 & 8 \\
\hline 6 & 49043205120000 & $45 \mathrm{~N}$ & $90 \mathrm{~W}$ & 15 & Pike Resources & 1-15 Federal & 6,100 & 6,120 & Frontier & shale & 0.97 & 1 \\
\hline 6 & 49043205120000 & $45 \mathrm{~N}$ & $90 \mathrm{~W}$ & 15 & Pike Resources & 1-15 Federal & 6,800 & 6,830 & Mowry & shale & 0.86 & 5 \\
\hline 6 & 49043205120000 & $45 \mathrm{~N}$ & $90 \mathrm{~W}$ & 15 & Pike Resources & 1-15 Federal & 7,040 & 7,060 & Thermopolis & shale & 0.93 & 7 \\
\hline 7 & 49017206620000 & $45 \mathrm{~N}$ & $99 \mathrm{~W}$ & 5 & Apache Corporation & 5-16 Federal & 3,180 & 3,210 & Cody & shale & 0.99 & 4 \\
\hline 7 & 49017206620000 & $45 \mathrm{~N}$ & $99 \mathrm{~W}$ & 5 & Apache Corporation & 5-16 Federal & 4,560 & 4,570 & Frontier & shale & 0.76 & 12 \\
\hline 7 & 49017206620000 & $45 \mathrm{~N}$ & $99 \mathrm{~W}$ & 5 & Apache Corporation & 5-16 Federal & 5,420 & 5,430 & Mowry & shale & 0.75 & 11 \\
\hline 7 & 49017206620000 & $45 \mathrm{~N}$ & $99 \mathrm{~W}$ & 5 & Apache Corporation & 5-16 Federal & 5,750 & 5,760 & Thermopolis & shale & 0.73 & 5 \\
\hline 8 & 49043201380000 & $46 \mathrm{~N}$ & $89 W$ & 6 & Chorney Oil & 2 Shoal Unit & 5,030 & 5,040 & Frontier & shale & 0.75 & 6 \\
\hline 8 & 49043201380000 & $46 \mathrm{~N}$ & $89 W$ & 6 & Chorney Oil & 2 Shoal Unit & 5,270 & 5,280 & Frontier & shale & 0.98 & 5 \\
\hline 8 & 49043201380000 & $46 \mathrm{~N}$ & $89 W$ & 6 & Chorney Oil & 2 Shoal Unit & 5,750 & 5,760 & Mowry & shale & 0.90 & 12 \\
\hline 8 & 49043201380000 & $46 \mathrm{~N}$ & $89 W$ & 6 & Chorney Oil & 2 Shoal Unit & 6,000 & 6,020 & Thermopolis & shale & 0.73 & 10 \\
\hline 9 & 49017060990000 & $46 \mathrm{~N}$ & $97 \mathrm{~W}$ & 29 & Midwest Oil & 1 Gywnn Ranch & 4,870 & 4,900 & Cody & shale & 0.98 & 9 \\
\hline 9 & 49017060990000 & $46 \mathrm{~N}$ & $97 \mathrm{~W}$ & 29 & Midwest Oil & 1 Gywnn Ranch & 6,230 & 6,250 & Frontier & shale & 1.07 & 9 \\
\hline 9 & 49017060990000 & $46 \mathrm{~N}$ & $97 \mathrm{~W}$ & 29 & Midwest Oil & 1 Gywnn Ranch & 6,950 & 6,970 & Mowry & shale & 0.97 & 12 \\
\hline 9 & 49017060990000 & $46 \mathrm{~N}$ & $97 \mathrm{~W}$ & 29 & Midwest Oil & 1 Gywnn Ranch & 7,250 & 7,280 & Thermopolis & shale & 1.05 & 8 \\
\hline 10 & 49043203090000 & $47 \mathrm{~N}$ & $94 \mathrm{~W}$ & 31 & National Coop & 1-31 Federal & 9,290 & 9,320 & Cody & shale & $\mathrm{nr}$ & \\
\hline 10 & 49043203090000 & $47 \mathrm{~N}$ & $94 \mathrm{~W}$ & 31 & National Coop & 1-31 Federal & 10,100 & 10,120 & Cody & shale & 1.16 & 14 \\
\hline 10 & 49043203090000 & $47 \mathrm{~N}$ & $94 \mathrm{~W}$ & 31 & National Coop & 1-31 Federal & 10,810 & 10,820 & Frontier & shale & 1.23 & 14 \\
\hline 10 & 49043203090000 & $47 \mathrm{~N}$ & $94 \mathrm{~W}$ & 31 & National Coop & 1-31 Federal & 11,460 & 11,480 & Mowry & shale & 1.36 & 12 \\
\hline 10 & 49043203090000 & $47 \mathrm{~N}$ & $94 \mathrm{~W}$ & 31 & National Coop & 1-31 Federal & 11,710 & 11,720 & Thermopolis & shale & 1.29 & 4 \\
\hline
\end{tabular}


Table 1. Vitrinite reflectance (Ro) data and locations for cuttings samples from wells drilled for oil and gas exploration and production in the Bighorn

Basin, north-central Wyoming and south-central Montana. Map no. refers to sample location numbers shown on figure 2.-Continued

[API, well number assigned by American Petroleum Institute; Tnsp., township; Rng., range; Sec., section; Lith., lithology; \%Ro, vitrinite reflectance measurement; n, number of readings per sample; nr, no reading. Queried (?) where uncertain. Depth is in feet.]

\begin{tabular}{|c|c|c|c|c|c|c|c|c|c|c|c|c|}
\hline $\begin{array}{c}\text { Map } \\
\text { no. }\end{array}$ & API & Tnsp. & Rng. & Sec. & Operator & Well & $\begin{array}{c}\text { Top } \\
\text { depth }\end{array}$ & $\begin{array}{c}\text { Bottom } \\
\text { depth }\end{array}$ & Formation & Lith. & $\% R_{0}$ & $\mathbf{n}$ \\
\hline 11 & 49029052770000 & $48 N$ & $101 W$ & 5 & Heep Oil & Rawhide Unit 2 & 700 & 710 & Cody & shale & $\mathrm{nr}$ & \\
\hline 11 & 49029052770000 & $48 N$ & $101 \mathrm{~W}$ & 5 & Heep Oil & Rawhide Unit 2 & 1,760 & 1,770 & Frontier & shale & 0.84 & 1 \\
\hline 11 & 49029052770000 & $48 N$ & $101 \mathrm{~W}$ & 5 & Heep Oil & Rawhide Unit 2 & 2,530 & 2,540 & Mowry & shale & 0.71 & 3 \\
\hline 11 & 49029052770000 & $48 N$ & $101 \mathrm{~W}$ & 5 & Heep Oil & Rawhide Unit 2 & 2,850 & 2,860 & Thermopolis & shale & 0.52 & 4 \\
\hline 12 & 49003051760000 & $49 N$ & $91 \mathrm{~W}$ & 15 & Chandler \& Simpson & 1 Empire & 580 & 600 & Frontier & shale & 0.37 & 8 \\
\hline 12 & 49003051760000 & $49 N$ & $91 \mathrm{~W}$ & 15 & Chandler \& Simpson & 1 Empire & 790 & 820 & Mowry & shale & 0.51 & 9 \\
\hline 12 & 49003051760000 & $49 N$ & $91 \mathrm{~W}$ & 15 & Chandler \& Simpson & 1 Empire & 1,000 & 1,030 & Mowry & shale & 0.56 & 8 \\
\hline 12 & 49003051760000 & $49 N$ & $91 \mathrm{~W}$ & 15 & Chandler \& Simpson & 1 Empire & 1,230 & 1,260 & Thermopolis & shale & $\mathrm{nr}$ & \\
\hline 12 & 49003051760000 & $49 N$ & $91 W$ & 15 & Chandler \& Simpson & 1 Empire & 1,430 & 1,450 & Cloverly & shale & 0.66 & 6 \\
\hline 13 & 49003204660000 & $49 N$ & $94 \mathrm{~W}$ & 11 & Excel Energy & 1-11 Dobie Creek & 8,550 & 8,570 & Cody & shale & 1.07 & 9 \\
\hline 13 & 49003204660000 & $49 N$ & $94 \mathrm{~W}$ & 11 & Excel Energy & 1-11 Dobie Creek & 9,790 & 9,820 & Frontier & shale & 1.12 & 7 \\
\hline 13 & 49003204660000 & $49 N$ & $94 \mathrm{~W}$ & 11 & Excel Energy & 1-11 Dobie Creek & 10,460 & 10,500 & Mowry & shale & 1.14 & 10 \\
\hline 13 & 49003204660000 & $49 N$ & $94 W$ & 11 & Excel Energy & 1-11 Dobie Creek & 10,730 & 10,740 & Thermopolis & shale & 1.21 & 7 \\
\hline 14 & 49029207390000 & $49 N$ & $100 \mathrm{~W}$ & 20 & Panther Exploration & 2 Bar TL & 4,380 & 4,410 & Cody & shale & 1.25 & 7 \\
\hline 14 & 49029207390000 & $49 N$ & $100 \mathrm{~W}$ & 20 & Panther Exploration & 2 Bar TL & 5,290 & 5,300 & Frontier & shale & 1.19 & 4 \\
\hline 14 & 49029207390000 & $49 N$ & $100 W$ & 20 & Panther Exploration & 2 Bar TL & 6,140 & 6,160 & Mowry & shale & 0.98 & 15 \\
\hline 14 & 49029207390000 & $49 N$ & $100 W$ & 20 & Panther Exploration & 2 Bar TL & 6,510 & 6,540 & Thermopolis & shale & 1.26 & 7 \\
\hline 15 & 49003055300000 & $51 \mathrm{~N}$ & $92 \mathrm{~W}$ & 2 & Amerada & 1 White Sheep & 540 & 550 & Cody & shale & 0.63 & 4 \\
\hline 15 & 49003055300000 & $51 \mathrm{~N}$ & $92 \mathrm{~W}$ & 2 & Amerada & 1 White Sheep & 1,070 & 1,080 & Frontier & shale & 0.64 & 16 \\
\hline 15 & 49003055300000 & $51 \mathrm{~N}$ & $92 \mathrm{~W}$ & 2 & Amerada & 1 White Sheep & 1,690 & 1,700 & Mowry & shale & 0.76 & 5 \\
\hline 15 & 49003055300000 & $51 \mathrm{~N}$ & $92 W$ & 2 & Amerada & 1 White Sheep & 1,960 & 1,970 & Thermopolis & shale & 0.78 & 1 \\
\hline
\end{tabular}


Table 1. Vitrinite reflectance (Ro) data and locations for cuttings samples from wells drilled for oil and gas exploration and production in the Bighorn

Basin, north-central Wyoming and south-central Montana. Map no. refers to sample location numbers shown on figure 2.-Continued

[API, well number assigned by American Petroleum Institute; Tnsp., township; Rng., range; Sec., section; Lith., lithology; \%Ro, vitrinite reflectance measurement; n, number of readings per sample; nr, no reading. Queried (?) where uncertain. Depth is in feet.]

\begin{tabular}{|c|c|c|c|c|c|c|c|c|c|c|c|c|}
\hline $\begin{array}{l}\text { Map } \\
\text { no. }\end{array}$ & API & Tnsp. & Rng. & Sec. & Operator & Well & $\begin{array}{l}\text { Top } \\
\text { depth }\end{array}$ & $\begin{array}{c}\text { Bottom } \\
\text { depth }\end{array}$ & Formation & Lith. & $\% R_{\circ}$ & $\mathbf{n}$ \\
\hline 16 & 49029210090000 & $51 \mathrm{~N}$ & 100W & 2 & Hunt/Impel & 1 Katrine Loch & 18,760 & 18,770 & Cody & shale & 1.86 & 7 \\
\hline 16 & 49029210090000 & $51 \mathrm{~N}$ & $100 \mathrm{~W}$ & 2 & Hunt/Impel & 1 Katrine Loch & 19,530 & 19,540 & Frontier & shale & 1.72 & 2 \\
\hline 16 & 49029210090000 & $51 \mathrm{~N}$ & $100 \mathrm{~W}$ & 2 & Hunt/Impel & 1 Katrine Loch & 20,300 & 20,310 & Mowry & shale & 2.10 & 11 \\
\hline 16 & 49029210090000 & $51 \mathrm{~N}$ & $100 \mathrm{~W}$ & 2 & Hunt/Impel & 1 Katrine Loch & 20,620 & 20,630 & Thermopolis & shale & 1.85 & 5 \\
\hline 17 & 49003203160000 & $52 \mathrm{~N}$ & $95 \mathrm{~W}$ & 26 & Gulf Oil & 1 Otto Federal & 9,950 & 9,960 & Cody & shale & 1.05 & 11 \\
\hline 17 & 49003203160000 & $52 \mathrm{~N}$ & $95 \mathrm{~W}$ & 26 & Gulf Oil & 1 Otto Federal & 11,330 & 11,360 & Frontier & shale & 1.34 & 10 \\
\hline 17 & 49003203160000 & $52 \mathrm{~N}$ & $95 \mathrm{~W}$ & 26 & Gulf Oil & 1 Otto Federal & 11,900 & 11,910 & Mowry & shale & 1.36 & 9 \\
\hline 17 & 49003203160000 & $52 \mathrm{~N}$ & $95 \mathrm{~W}$ & 26 & Gulf Oil & 1 Otto Federal & 12,140 & 12,150 & Thermopolis & shale & 1.43 & 15 \\
\hline 18 & 49003057770000 & $53 \mathrm{~N}$ & $96 \mathrm{~W}$ & 1 & Sinclair Oil \& Gas & 1 Govt. & 9,200 & 9,220 & Cody & shale & 0.55 & 21 \\
\hline 18 & 49003057770000 & $53 \mathrm{~N}$ & $96 \mathrm{~W}$ & 1 & Sinclair Oil \& Gas & 1 Govt. & 10,250 & 10,280 & Frontier & shale & 1.52 & 12 \\
\hline 18 & 49003057770000 & $53 \mathrm{~N}$ & $96 \mathrm{~W}$ & 1 & Sinclair Oil \& Gas & 1 Govt. & 10,940 & 10,970 & Mowry & shale & 1.23 & 20 \\
\hline 18 & 49003057770000 & $53 \mathrm{~N}$ & $96 \mathrm{~W}$ & 1 & Sinclair Oil \& Gas & 1 Govt. & 11,270 & 11,290 & Thermopolis & shale & 1.67 & 14 \\
\hline 19 & 49029058940000 & $55 \mathrm{~N}$ & $99 \mathrm{~W}$ & 3 & Tidewater Oil & 1 Atteberry & 8,520 & 8,540 & Cody & shale & 1.26 & 6 \\
\hline 19 & 49029058940000 & $55 \mathrm{~N}$ & $99 \mathrm{~W}$ & 3 & Tidewater Oil & 1 Atteberry & 9,500 & 9,510 & Frontier & shale & 1.00 & 7 \\
\hline 19 & 49029058940000 & $55 \mathrm{~N}$ & $99 \mathrm{~W}$ & 3 & Tidewater Oil & 1 Atteberry & 10,450 & 10,470 & Mowry & shale & 1.30 & 4 \\
\hline 19 & 49029058940000 & $55 \mathrm{~N}$ & $99 \mathrm{~W}$ & 3 & Tidewater Oil & 1 Atteberry & 10,700 & 10,710 & Thermopolis & shale & 1.26 & 8 \\
\hline 20 & 49029059970000 & $56 \mathrm{~N}$ & $98 \mathrm{~W}$ & 1 & C.E. Brehm & 1 Deaton Govt. & 2,840 & 2,860 & Frontier & shale & 0.63 & 3 \\
\hline 20 & 49029059970000 & $56 \mathrm{~N}$ & $98 \mathrm{~W}$ & 1 & C.E. Brehm & 1 Deaton Govt. & 3,240 & 3,260 & Frontier & shale & 0.63 & 11 \\
\hline 20 & 49029059970000 & $56 \mathrm{~N}$ & $98 \mathrm{~W}$ & 1 & C.E. Brehm & 1 Deaton Govt. & 3,780 & 3,800 & Mowry & shale & 0.53 & 6 \\
\hline 20 & 49029059970000 & $56 \mathrm{~N}$ & $98 \mathrm{~W}$ & 1 & C.E. Brehm & 1 Deaton Govt. & 4,170 & 4,200 & Thermopolis & shale & 0.90 & 6 \\
\hline
\end{tabular}


Table 1. Vitrinite reflectance (Ro) data and locations for cuttings samples from wells drilled for oil and gas exploration and production in the Bighorn

Basin, north-central Wyoming and south-central Montana. Map no. refers to sample location numbers shown on figure 2.-Continued

[API, well number assigned by American Petroleum Institute; Tnsp., township; Rng., range; Sec., section; Lith., lithology; \%Ro, vitrinite reflectance measurement; n, number of readings per sample; nr, no reading. Queried (?) where uncertain. Depth is in feet.]

\begin{tabular}{|c|c|c|c|c|c|c|c|c|c|c|c|c|}
\hline $\begin{array}{l}\text { Map } \\
\text { no. }\end{array}$ & API & Tnsp. & Rng. & Sec. & Operator & Well & $\begin{array}{c}\text { Top } \\
\text { depth }\end{array}$ & $\begin{array}{l}\text { Bottom } \\
\text { depth }\end{array}$ & Formation & Lith. & $\% R_{0}$ & $\mathbf{n}$ \\
\hline 21 & 49003205680000 & $57 \mathrm{~N}$ & $96 \mathrm{~W}$ & 27 & Forest Oil & 1 Stevens & 830 & 840 & Mowry & shale & 0.58 & 7 \\
\hline 21 & 49003205680000 & $57 \mathrm{~N}$ & $96 \mathrm{~W}$ & 27 & Forest Oil & 1 Stevens & 1,100 & 1,130 & Mowry & shale & 0.61 & 4 \\
\hline 21 & 49003205680000 & $57 \mathrm{~N}$ & $96 \mathrm{~W}$ & 27 & Forest Oil & 1 Stevens & 1,400 & 1,430 & Thermopolis & shale & 0.66 & 7 \\
\hline 21 & 49003205680000 & $57 \mathrm{~N}$ & $96 \mathrm{~W}$ & 27 & Forest Oil & 1 Stevens & 1,550 & 1,560 & Cloverly & shale & $\mathrm{nr}$ & \\
\hline 22 & 49029065930000 & $58 N$ & $98 \mathrm{~W}$ & 23 & Continental & 6 Pacific & 900 & 930 & Frontier & shale & $\mathrm{nr}$ & \\
\hline 22 & 49029065930000 & $58 N$ & $98 \mathrm{~W}$ & 23 & Continental & 6 Pacific & 1,090 & 1,110 & Mowry & shale & 0.67 & 2 \\
\hline 22 & 49029065930000 & $58 N$ & $98 \mathrm{~W}$ & 23 & Continental & 6 Pacific & 1,230 & 1,250 & Mowry & shale & 0.80 & 10 \\
\hline 23 & 25009051910000 & $7 S$ & $21 \mathrm{E}$ & 3 & Ohio Oil Company & 18 N.P. & 2,900 & 2,910 & $\begin{array}{c}\text { lower } \\
\text { Mesaverde }\end{array}$ & coal & 0.60 & 25 \\
\hline 23 & 25009051910000 & $7 S$ & $21 \mathrm{E}$ & 3 & Ohio Oil Company & 18 N.P. & 3,520 & 3,570 & Cody & coal? & 0.55 & 25 \\
\hline 23 & 25009051910000 & $7 S$ & $21 \mathrm{E}$ & 3 & Ohio Oil Company & 18 N.P. & 4,120 & 4,155 & Frontier & coal/carb & 0.55 & 21 \\
\hline 23 & 25009051910000 & $7 S$ & $21 \mathrm{E}$ & 3 & Ohio Oil Company & 18 N.P. & 4,990 & 5,010 & Mowry & shale & 0.58 & 2 \\
\hline 23 & 25009051910000 & $7 S$ & $21 E$ & 3 & Ohio Oil Company & 18 N.P. & 5,360 & 5,380 & Thermopolis & shale & 0.54 & 25 \\
\hline 24 & 25009211550000 & $8 S$ & $23 \mathrm{E}$ & 9 & Arco & 1 Hunt Creek & 2,610 & 2,640 & $\begin{array}{c}\text { lower } \\
\text { Mesaverde }\end{array}$ & coal & 0.54 & 11 \\
\hline 24 & 25009211550000 & $8 S$ & $23 \mathrm{E}$ & 9 & Arco & 1 Hunt Creek & 3,600 & 3,630 & Cody & shale & 0.87 & 4 \\
\hline 24 & 25009211550000 & $8 S$ & $23 E$ & 9 & Arco & 1 Hunt Creek & 4,500 & 4,520 & Frontier & coal/carb & 0.55 & 27 \\
\hline 24 & 25009211550000 & $8 S$ & $23 \mathrm{E}$ & 9 & Arco & 1 Hunt Creek & 5,310 & 5,330 & Mowry & shale & 0.74 & 6 \\
\hline 24 & 25009211550000 & $8 S$ & $23 E$ & 9 & Arco & 1 Hunt Creek & 5,730 & 5,750 & Thermopolis & shale & 0.87 & 4 \\
\hline
\end{tabular}

